\title{
Bilateral olfaction: two is better than one for navigation Baranidharan Raman* ${ }^{* \dagger}$, Iori Ito* and Mark Stopfer*
}

\author{
Addresses: *National Institute of Child Health and Human Development, NIH, Lincoln Drive, Bethesda, MD 20892, USA. \\ ${ }^{\dagger}$ National Institute of Standards and Technology, Gaithersburg, MD 20899, USA. \\ Correspondence: Mark Stopfer. Email: stopferm@mail.nih.gov
}

Published: 3I March 2008

Genome Biology 2008, 9:212 (doi: 10.1 I86/gb-2008-9-3-2।2)

The electronic version of this article is the complete one and can be found online at http://genomebiology.com/2008/9/3/2/2

(c) 2008 BioMed Central Ltd

\begin{abstract}
Do animals require bilateral input to track odors? A recent study reveals that fruit fly larvae can localize odor sources using unilateral inputs from a single functional sensory neuron, but that an enhanced signal-to-noise ratio provided by dual inputs is helpful in more challenging environments.
\end{abstract}

Biological sensory systems often make use of asymmetries in sensory inputs to extract information about the environment. The visual system, for example, exploits disparities in the two-dimensional images obtained from the left and the right eyes to extract information about depth [1]. The auditory system uses the phase and intensity differences of stereo inputs to localize sound sources [2]. Relatively little is known about the importance of bilateral inputs in olfaction. Our own noses feature twin nostrils; insects have paired antennae. What advantages do such configurations provide? A recent study by Louis and colleagues [3] examined the significance of paired inputs for odor navigation in an animal offering numerous experimental advantages, the larva of the fruit fly, Drosophila melanogaster.

Studying how animals carry out chemotaxis, that is, how they navigate through chemical gradients, requires careful behavioral assays conducted within well-controlled spatial distributions of chemicals. In the case of olfaction, it is a significant technical challenge to generate the stable odor gradients needed for such a study. Louis et al. [3] developed a novel and clever approach: they built a small test chamber whose ceiling, an inverted 96-well plate, suspended an ordered array of droplets of sequentially diluted odorants (Figure 1). The authors confirmed that this array generated the desired airborne odor gradient within the test chamber by means of Fourier transformed infrared spectroscopy. Equipped with this well-controlled stimulus field, the authors set about examining chemotaxis in fruit fly larvae.
In the larva, the transduction of chemical stimuli into neural representations begins in two dorsally located olfactory organs that are about 100 micrometers apart. Each olfactory organ normally contains 21 sensory neurons, each expressing one or two receptor genes together with the universally coexpressed $O R 83 b$ gene [4]. Earlier studies by the authors had established that knocking out the $O R 83 \mathrm{~b}$ coreceptor gene removes essentially all odor-driven behavior in these larvae [4]. By randomly rescuing the co-receptor gene in either the left or the right olfactory organ in transgenic $O R 83 b$ knockout preparations, the authors generated unilateral animals - perfect for answering interesting questions about bilateral chemoreception.

\section{Is one just as good as two?}

Do the larvae require a full complement of receptors to reliably locate odor sources? Surprisingly, transgenic larvae with unilateral input from a single olfactory neuron were able to locate odor sources just as well as wild-type larvae. In fact, bilateral transgenic larvae with a single functional receptor neuron in each of their olfactory organs actually showed greater odor sensitivity than wild-type larvae. This apparently odd result may point toward an odor-coding scheme in the wild type in which ensembles of sensors with a low signal-to-noise ratio are combined with inputs with a high signal-to-noise ratio. Or, alternatively, in the wild type, competition among downstream neurons driven by different receptor neurons could diminish overall 
(a) Chamber for behavioral tests

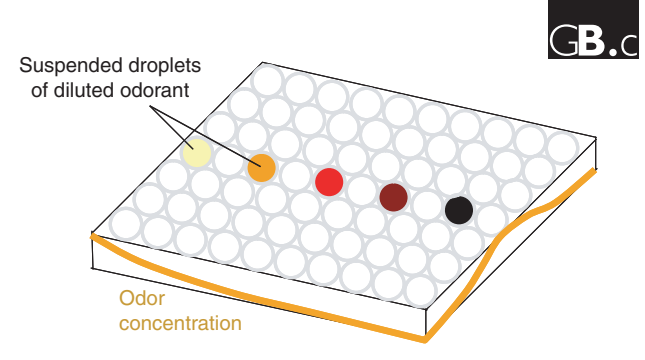

(b) Chemotaxing larvae ascend along odor gradients

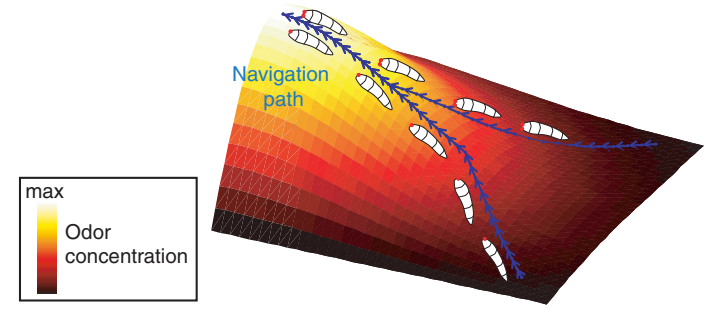

Figure I

Investigating chemotaxis by Drosophila larvae. (a) Louis et al. [3] generated a well-structured airborne odor concentration gradient by suspending droplets of odorant at different concentrations from the ceiling of their test chamber (yellow denotes the highest concentration; black the lowest). The arrangement of droplets generated a spatial concentration distribution that varies from one end of the chamber to the other and from the middle of the chamber (high) to the sides. (b) Both unilateral and bilateral transgenic larvae navigate odor fields by detecting local concentration gradients. By moving along the direction of the steepest intensity variation, the larvae reliably locate the source of the odor.

sensitivity. Schemes like these may function to promote odor discrimination, another task mediated by the same circuitry.

The authors found that both transgenic and wild-type larvae navigate by constantly orienting themselves along the direction of the steepest local concentration gradient (Figure 1). The larval rate of turning was greatest in low-concentration regions and decreased as the larvae progressed towards the concentration peak. This 'direct chemotaxis' is strikingly different from the 'biased random walk' strategy used by bacteria, which change direction at random, but alter the intervals between turns to bias movement toward attractants and away from repellants [5].

Interestingly, the authors noticed a side-dependent bias in the unilateral animals. Both left- and right-sided animals have a single functional receptor neuron, yet right-sided larvae performed chemotaxis significantly better than their left-sided counterparts. In larvae (unlike in adult flies) sensory inputs from each side remain segregated throughout the peripheral olfactory pathway. Thus, the observed right-side bias suggests disparities in downstream processing. This inherent right-side bias is not unique to these larvae - lateralization of olfactory processing has also been reported in a few other invertebrate species [6]. The importance of this bias for odor processing and olfactory behavior remains unclear.

The two olfactory organs are so close together in fruit fly larvae that any odor concentration differences between them would be undetectably slight, and so it seems unlikely that bilateral concentration comparisons could provide useful cues for successful navigation. So how do these organisms locate odor sources? The most likely possibility is that the larvae use a mechanism that allows comparisons between at least two consecutive concentration measurements made over time. Thus, the results from Louis et al. [3] suggest that a form of working memory of the concentration of recent samples is required for chemotaxis by Drosophila larvae.

\section{Navigating complex environments}

Why then have two separate olfactory organs? The authors found that fruit fly larvae with dual inputs performed significantly better than their unilateral counterparts when challenged to navigate through complex odor environments with shallow, linear gradients and high offset concentrations. How do bilateral inputs aid with chemotaxis? It was not the case that simply doubling olfactory input lowered olfactory response thresholds, as the lowest concentration in the behavioral assay was above the detection level of the unilateral animals. Louis et al. [3] note that, theoretically, integrating information from $n$ redundant sensors can result in $\sqrt{n}$ times enhancement in the signal-to-noise ratio, provided the noise in the separate sensors remains uncorrelated. Hence, the bilateral larvae should possess a lower detection level and an ability to make concentration measurements with a resolution at most $\sqrt{2}$ times better than the unilateral animals (Figure 2). Perhaps two physically separated olfactory organs provide inputs that are less noisecorrelated than inputs from a single receptor organ. The observed improvement in performance may be due to an improved signal-to-noise ratio provided by the neural integration of redundant sensory information, or to a nonlinear process of lateralized bilateral inputs in the central brain, or to both.

Adult flies may use a different strategy. Unlike larvae, in adults around 10-40 receptor neurons of the same type are present in each antenna and project bilaterally to both left and right antennal lobes. Hence, in the adult, integration of redundant inputs begins at a very early stage in olfactory processing. Whether this unique wiring scheme enhances the spatial comparison of simultaneous bilateral inputs [7] or only increases the number of redundant receptors and, therefore, the signal-to-noise ratio, remains unknown. 
(a)

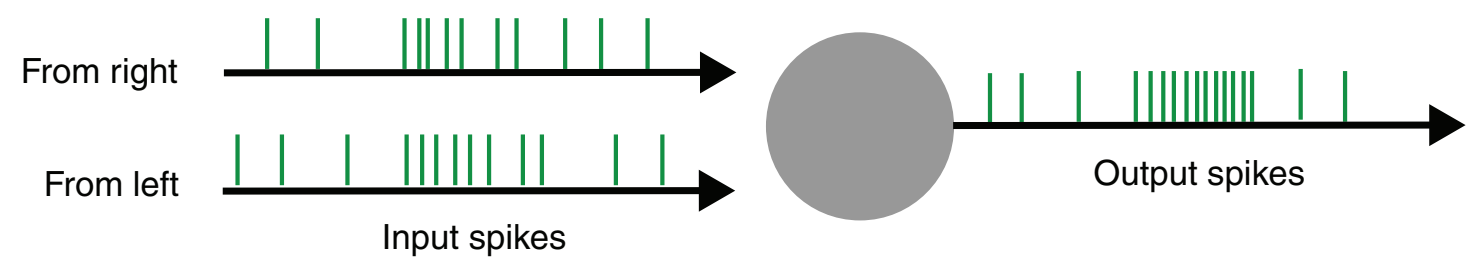

(b)

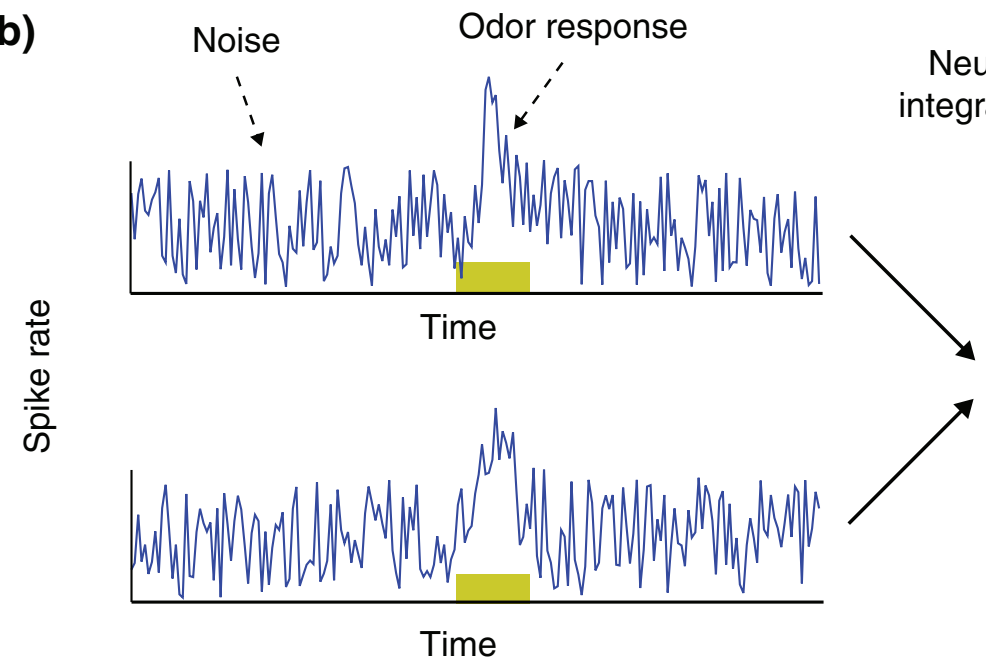

Neural

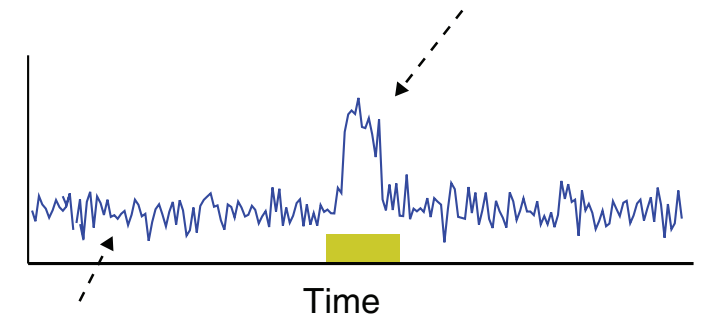

Less noise

Figure 2

Neural integration of bilateral olfactory inputs enhances signal-to-noise ratio. (a) Schematic diagram of the bilateral olfactory input pathways and a hypothetical central neuron (grey circle) receiving those inputs. Information is transmitted as spiking activity. Typically, in the absence of any olfactory stimulus, the receptor neurons tend to show a baseline spiking response that contributes to the 'noise' in the system. Both the detection level and the measurement resolution of the system are dependent on the input noise level. (b) Neural integration can reduce uncorrelated noise. The plots on the left represent the firing rate of two receptor neurons over time. The baseline fluctuations observed in the two independent channels (left) are reduced after integrating them (right), thus improving signal-to-noise ratio. This improvement may be the chief contribution of dual olfactory inputs to chemotaxis. The green box indicates the release of a puff of odor.

Stereo olfactory cues are more important for humans and other animals with olfactory organs that are well separated in space $[8,9]$. Humans, for example, can track odors based on comparisons of concentration measurements made over time alone, but also use inter-nostril concentration differences to improve tracking performance: occluding one nostril or providing the same odor information to both nostrils significantly reduces a person's ability to locate odor sources quickly [9].

As shown by Louis and colleagues [3], Drosophila, with its simple brain structure and wealth of genetic tools, provides a useful system for the study of olfaction and odor-evoked behavior. It will be interesting to determine the role of bilateral inputs in adult flies and compare their navigation strategies with those of the larvae. And it will be especially interesting to explore the significance and neural basis of the transient, working memory processes apparently needed to mediate chemotaxis. The use of genetically manipulated flies and their larvae will no doubt contribute greatly to these efforts [10].

\section{References}

I. Ohzawa I, DeAngelis GC, Freeman RD: The neural coding of stereoscopic depth. NeuroReport 1997, 8:iii-xii.

2. King AJ, Schnupp JWH, Doubell, TP: The shape of ears to come: dynamic coding of auditory space. Trends Cogn Sci 200 I, 5:26I-270.

3. Louis M, Huber T, Benton R, Sakmar TP, Vosshall LB: Bilateral sensory input enhances chemotaxis. Nature Neurosci 2008, II:187-199.

4. Larsson MC, Domingos Al, Jones WD, Chiappe ME, Amrein $\mathrm{H}$ and Vosshall LB: Or83b encodes a broadly expressed odorant receptor essential for Drosophila olfaction. Neuron 2004, 43:703-7I4. 
5. Berg HC: Motile behavior of bacteria. Physics Today 2000, 53:24-29.

6. Letzkus P, Ribi W, Wood J, Zhu H, Zhang S, Srinivasan M: Lateralization of olfaction in the honeybee Apis mellifera. Curr Biol 2006, 16:147I-I476.

7. Rodrigues $\mathrm{V}$ : Spatial coding of olfactory information in the antennal lobe of Drosophila melanogaster. Brain Res 1988, 453:299-307.

8. Raghan R, Clement JP, Bhalla US: Rats smell in stereo. Science 2006, 3II:666-670.

9. Porter J, Craven B, Khan RM, Chang S, Kang I, Judkewitz B, Volpe J, Settles G, Sobel N: Mechanisms for scent tracking in humans. Nature Neurosci 2007, 10:27-29.

10. Keene AC, Waddell S: Drosophila olfactory memory: single genes to complex neural circuits. Nat Rev Neurosci 2007, 8:34 I-354. 